Journal of Engineering and Applied Sciences 15 (2): 694-697, 2020

ISSN: 1816-949X

(C) Medwell Journals, 2020

\title{
Rheological and Filtration Parameters of the Polymer Salt Drilling Fluids Based on Xanthan Gum
}

\author{
Pavel Aleksandrovich Blinov and Mikhail Vladimirovich Dvoynikov \\ Department of Borehole Drilling, Oil and Gas Faculty, Saint-Petersburg Mining University, \\ 21st Line V.O., 2, 199106 Saint Petersburg, Russia
}

\begin{abstract}
When drilling for oil and gas there are different physical and chemical processes. This is the result of the impact of drilling mud with rocks. This can be diffusion, filtration, heat transfer, capillary flow, etc. Filtrationis one of the most important processes that affect drilling operations. It affects the absorption of drilling fluid, the manifestation of fluids, the formation of clay cake on the walls of the hole and the emergence of other phenomena. When calling the influx phenomenon can be observed that determine the quality and efficiency of injection wells.
\end{abstract}

Key words: Drilling fluid, drilling mud, polymer salt drilling fluids, rheology and filtration of drilling mud, capillary flow, absorption

\section{INTRODUCTION}

At the current stage of development of technics and technology the bulk of the drilling is under conditions of excess bottom hole pressure over the formation, i.e., formation overburden in accordance with the requirements of the applicable safety regulations in the oil and gas industry (Ovchinnikov, 2010). The disadvantages include (Ryazanov, 2005).

The decline in filtration-capacitive properties of rocks composing the collector in the near-well zone due to the penetration of the disperse phase and the dispersive environment of drilling fluids. Absorption of drilling fluids under the action of differential pressure acting from the side of the wellbore. The potential for stuck pipe drill pipe caused by differential pressure acting on the borehole wall against intervals of permeable rocks. The decrease in the mechanical speed of destruction of rocks on the bottom of the well, caused by the excess pressure of the mud column.

From the condition in which it is located in the downhole area of a formation depends on the effectiveness of methods of development of oil and gas. When the reduced initial permeability in borehole zone of reservoir it is recommended to use different methods of development. The safety zone during the arrangement of wells is an important and urgent task.

Well productive formation is a nonequilibrium system. The changes occurring in it are determined by the ratio of the pressure, temperature, drilling fluid properties and heterogeneity of formations, characteristics of rocks, reservoirs and fluids which are saturated. The reservoir characteristics are subjected to different changes at autopsy a perforation, drilling, stimulation and testing of wells. They can increase by 1-2 orders of magnitude and completely stop fluid flow. Termination, in particular intensification of focus on ways to improve the quality characteristics of the reservoirs and increase well productivity (Ovchinnikov, 2010).

The efficiency of the development and exploration of gas and oil depends on the quality characteristics of the reservoirs in the borehole zone.

As a result of the analysis of field and experimental data confirmed the negative effect of the concentration of the solid fraction of drilling mud to the natural reservoir characteristics of the permeable formations. When the solid phase penetrates the pores in particular in cracks collectors, greatly reduced the permeability of the near wellbore zone. The filling conductive paste of clay with a mass of high density, it for a long time clogs the channels for transferring hydrocarbons. Colloidal particles penetrate into the formation are adsorbed in the pores of the collector. Most often this occurs at the boundary between gas (oil) and the filtrate, freedom of movement is lost. The permeability of the reservoir is greatly reduced due to the interfacial membrane and adsorption layers which reduce the cross section of the pore channels.

Studying the effect of drilling fluids which are used today in order to open productive formations, it is worth emphasizing that the reservoir properties deteriorate completely from all available solutions (Blinov and Dvoynikov, 2016).

To reduce the negative impact on the permeability of the borehole formation areas exclude solid phase of drilling fluids. Also, increase the speed of drilling which

Corresponding Author: Pavel Aleksandrovich Blinov, Department of Borehole Drilling, Oil and Gas Faculty, Saint-Petersburg Mining University, 21st Line V.O., 2, 199106 Saint Petersburg, Russia 
is an important factor when using systems containing no solid phase. Reduced drilling time distances of the productive horizon, the period of the interaction solution for drilling reservoir rocks, resulting in a minimized negative impact on the filtration and capacitive characteristics.

The dispersive environment of drilling fluid, circulating in the area of the productive formation wells under the influence of pressure fluctuations are filtered into permeable porous strata. The filtrate is the water containing the chemicals in dissolved state. Most often, the filtrate penetrating the layers, impairing their filtration properties and helps to reduce the production wells.

The main advantage of high-molecular linear polymers is that they are capable of forming a thin film on the surface of the mineral. Preserving the mechanical integrity of the shale which has been softened under the action of the drilling fluid.

Xanthan gum a natural chemical compound $\left(\mathrm{C}_{35} \mathrm{H}_{49} \mathrm{O}_{29}\right)_{\mathrm{n}}$ food additive E415, belongs to the group of the stabilizers. Xanthan is a powder of white or zerog colour, odour and taste. The substance is very soluble in water does not lose its properties in a wide range of acidity and temperature (Kozak, 2006). Xanthan gum is used to regulate the rheological properties of the fluids while drilling based on water. It gives the possibility to increase the viscosity of solution even at low concentration, the gum provides a retaining and carrying characteristics.

This reagent has a particular rheological profile. Under strong shear stress, it allows the solutions to flow freely at the same time the gum at a low flow rate converts the solution into an elastic gel. Such solutions are called solutions of the inverse viscosity, high flow solution pressure loss minimized at the same time at low speeds is ensured by the high holding ability. Salt solutions ( $\mathrm{NaCl}, \mathrm{KCl}, \mathrm{CaCl}_{2}, \mathrm{MgCl}_{2}$ ) are used extensively as water treatment agents in such cases:

- $\quad$ Drilling occurs in perennial permafrost

- $\quad$ The drilling takes place in a suspended salts

- $\quad$ The liquid is used as the silencer of wells workover

- When the plugging of wells as a buffer fluid

- Salt solutions on the basis of water capable of preventing the swelling of rocks containing clay

The high content of clay in the rocks of the productive formation requires special techniques for its opening. To eliminate the swelling of these rocks, actively using special solutions that have additives in the form of mineral salts. Thanks to the active substances prevents the swelling of shale.

The main advantage of high-molecular linear polymers is that they are capable of forming a thin film on the surface of the mineral. Preserving the mechanical integrity of the shale which has been softened under the action of the drilling fluid.

Xanthan gum a natural chemical compound $\left(\mathrm{C}_{35} \mathrm{H}_{49} \mathrm{O}_{29}\right)_{\mathrm{n}}$ food additive E415, belongs to the group of the stabilizers. Xanthan is a powder of white or zerogcolour, odour and taste. The substance is very soluble in water does not lose its properties in a wide range of acidity and temperature.

Xanthan gum is used to regulate the rheological properties of the fluids while drilling based on water. It gives the possibility to increase the viscosity of solution even at low concentration, the gum provides a retaining and carrying characteristics.

This reagent has a particular rheological profile. Under strong shear stress, it allows the solutions to flow freely, at the same time the gum at a low flow rate converts the solution into an elastic gel. Such solutions are called solutions of the inverse viscosity, high flow solution pressure loss minimized at the same time at low speeds is ensured by the high holding ability.

Biopolymer drilling fluids have a lower density than clay in addition, they allow, under unbalanced pressure on the bottom and a controlled release of formation fluid to drill at maximum speed (Ptichkin and Ptichkin, 2005).

To determine the filtration and rheological properties of biopolymer salt drilling fluids studies have been conducted. As used biopolymer is xanthan gum. As salts used chlorides of sodium $(\mathrm{NaCl})$, potassium $(\mathrm{KCl})$, Magnesium $\left(\mathrm{MgCl}_{2}\right)$ and Calcium $\left(\mathrm{CaCl}_{2}\right)$.

\section{MATERIALS AND METHODS}

The parameters of drilling fluids was determined by the method API 13B. Filtering was determined on the filter press Fann HTHP. Rheological properties were investigated on the instrument Fann 35 SA. The tests were carried out at room temperature.

\section{RESULTS AND DISCUSSION}

To select the optimal composition of the biopolymer were investigated solutions with a concentration of $0.3-0.8 \%$. The rheological curves are presented in Fig. 1.

With the increase in concentration of xanthan gum increases the Plastic Viscosity (PV) and Yield Point (YP) (Table 1). The optimal concentration is $0.5 \%$. This concentration will be used next.

Since, many polymers are not very well behaved in the presence of salts conducted the following tests. For a research of influence of concentration of salts their rheological parameters were defined (Fig. 2 and 3). 
Table 1: The rheological properties of solutions of xanthan gum with a concentration of $0.3-0.8 \%$

\begin{tabular}{|c|c|c|c|c|c|c|}
\hline \multirow[b]{2}{*}{ Rheological properties } & \multicolumn{6}{|c|}{ Concentration of xanthan gum (\%) } \\
\hline & 0.3 & 0.4 & 0.5 & 0.6 & 0.7 & 0.8 \\
\hline Plastic Viscosity (PV) (MPa) & 7.5 & 9.2 & 10.50 & 12.0 & 13.5 & 15.0 \\
\hline Yield Point (YP) (Pa) & 14.0 & 21.6 & 29.50 & 38.0 & 47.5 & 57.0 \\
\hline Apparent Viscosity (AV) (MPa) & 14.5 & 20.0 & 25.25 & 31.0 & 37.2 & 43.5 \\
\hline YP/PV & 1.9 & 2.4 & 2.80 & 3.2 & 3.5 & 3.8 \\
\hline Consistency index (K) & 7.4 & 15.0 & 25.10 & 36.9 & 51.6 & 67.0 \\
\hline Behavior index (n) & 0.4 & 0.4 & 0.30 & 0.3 & 0.3 & 0.3 \\
\hline
\end{tabular}

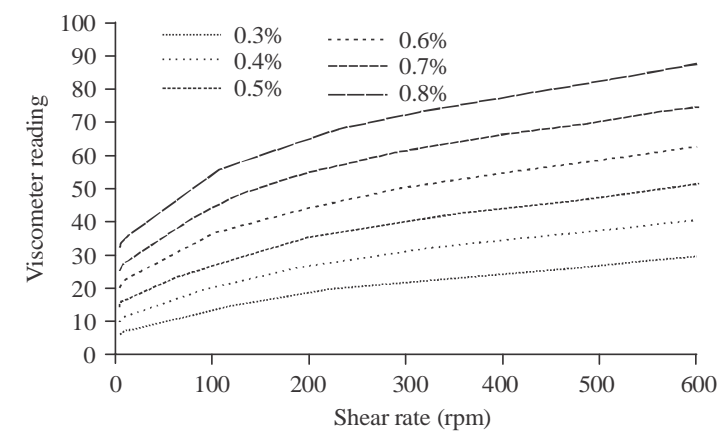

Fig. 1: Rheology of biopolymer solutions of xanthan gum with a concentration of $0.3-0.8 \%$

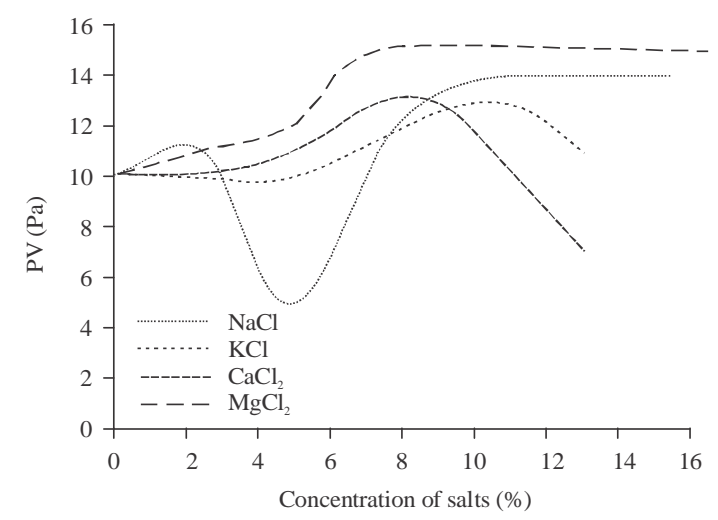

Fig. 2: The dependence of Plastic Viscosity (PV) on the salt concentration of the polymer solution of $0.5 \%$ xanthan gum

As can be seen from the graphs in Fig. 2 and 3 the dependences are not linear. They have extrema which are related to changes of the spatial structure of the polymer. This pronounced dependence of the sodium chloride. Up to $2.5 \%$ based on the primary structure of the polymer and the growth of the plastic viscosity. Then at a concentration of $5 \%$ the formation of secondary spatial structure has a lower plastic viscosity and shear stress. After that, until a concentration of $8 \%$ is the formation of tertiary spatial structure. This is evident in the extremum YP. Further, increase in concentration leads, most likely to coagulation of the polymer. This is clearly seen on the example of calcium chloride which YP $=0$ at a concentration of $13 \%$ and PV decreases dramatically.

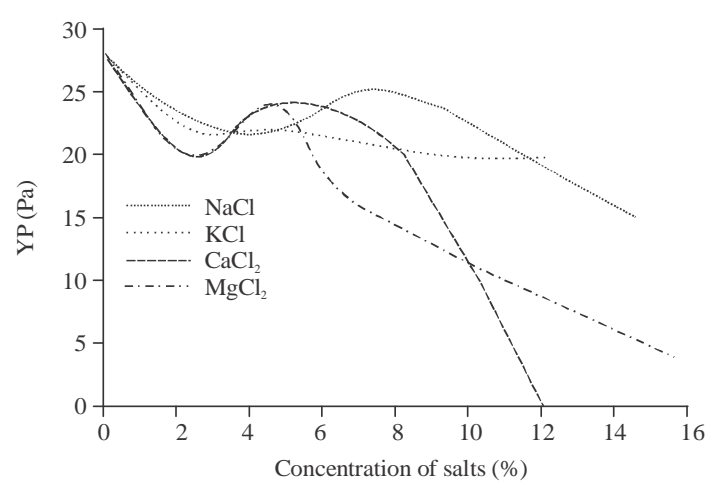

Fig. 3: The dependence of Yield Point (YP) on the salt concentration of the polymer solution of $0.5 \%$ xanthan gum

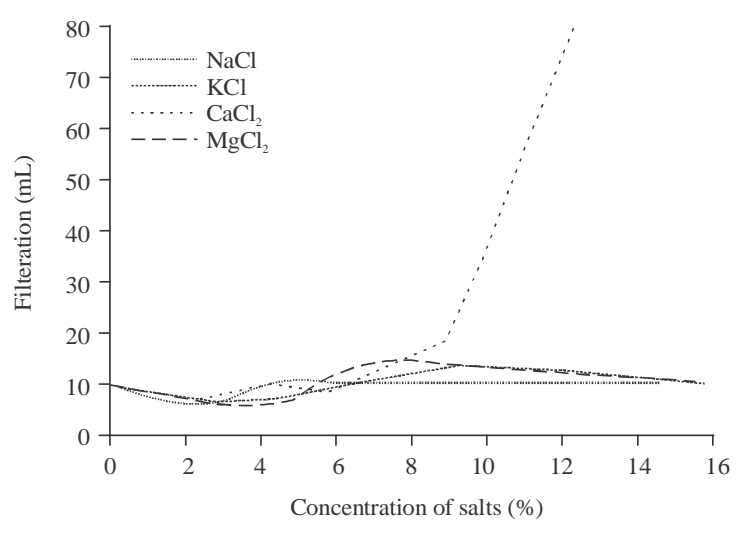

Fig. 4: The dependence of filtration on the salt concentration of the polymer solution of $0.5 \%$ xanthan gum

Similar dependencies are traced and the use of other salts. To confirm this, studies have been conducted filtration of these solutions (Fig. 4).

The study of drilling fluids filtration showed a similar result. This proves that the polymer in the solution changes its spatial structure from primary to tertiary. In addition, it is seen that the calcium chloride loses its structure and begins to coagulate.

When added to polymer drilling mud chloride, the polymer changes its structure from primary to secondary and then to tertiary. After that, the polymer may coagulate as for example, calcium chloride. 
Scope of polymer and salt solutions based on xanthan gum and chloride could be next. The concentration of sodium chloride in the polymer solution should not exceed $8 \%$ because of a reduction in YP. When using potassium chloride concentration should be not more than $10.5 \%$ due to the decrease of rheological and filtration parameters. Magnesium chloride and calcium chloride can be applied to a concentration of $7-8 \%$.

Further studies of polymer salt solutions should be focused on the study of their inhibiting properties which are very important when drilling in clay rocks.

\section{CONCLUSION}

To create and scientifically justify methods to achieve the results of improving the efficiency of opening oil and gas reservoirs which can prevent various complications, you need to own set of rules, laws and dependencies on the basis of which the movement of gases and liquids in the formations. This study is the object of the theory of filtration.

\section{REFERENCES}

Blinov, P.A. and M.V. Dvoynikov, 2016. The process of hardening loose rock by Mud filtrat. Intl. J. Appl. Eng. Res., 11: 6630-6632.

Kozak, N., 2006. Polysaccharide Xanthan: Properties and potential of application. Polym. Money, Vol. 2006, No. 1.

Ovchinnikov, V.P., 2010. Completions. Tyumen State Oil and Gas University, Tyumen, Russia, Pages: 451.

Ptichkin, I.I. and N.M. Ptichkin, 2005. Food Polysaccharides: Structural Levels and Functionality. Publishing House FGOU, Saratov, Russia, Pages: 164.

Ryazanov, A.Y., 2005. Encyclopedia of Drilling Fluids. Chronicle Publishing Company, Orenburg, Russia, Pages: 664 . 